PROCEEDINGS OF THE

AMERICAN MATHEMATICAL SOCIETY

Volume 129, Number 12, Pages 3543-3548

S 0002-9939(01)06261-X

Article electronically published on June 13, 2001

\title{
LARGE SETS OF ZERO ANALYTIC CAPACITY
}

\author{
JOHN GARNETT AND STAN YOSHINOBU \\ (Communicated by Juha M. Heinonen)
}

\begin{abstract}
We prove that certain Cantor sets with non-sigma-finite onedimensional Hausdorff measure have zero analytic capacity.
\end{abstract}

\section{Introduction}

In this paper we consider a Cantor set $K$ similar to the $\frac{1}{4}$-Cantor set of [G70 and [184]. Fix $p>2$ and for $n>0$ define

$$
\sigma_{n}=4^{-n} a_{n}=4^{-n}[\log (n+1)]^{1 / p} .
$$

Set $K_{0}=[0,1] \times[0,1]$ and $K_{1}=\bigcup_{j=1}^{4} K_{1, j}$, where $K_{1, j} \subset K_{0}$ is a square of sidelength $\sigma_{1}$ having sides parallel to the axis and containing one of the four corners of $K_{0}$. Next take $4^{2}$ squares $K_{2, j}$ of sidelength $\sigma_{2}$, one in each corner of each square $K_{1, j}$, and define $K_{2}=\bigcup_{j=1}^{4^{2}} K_{2, j}$. Continuing we obtain $K_{n}=\bigcup_{j=1}^{4^{n}} K_{n, j}$, where $K_{n, j}$ is a square of sidelength $\sigma_{n}$. The Cantor set we study is

$$
K=K(p)=\bigcap_{n=1}^{\infty} K_{n} .
$$

If $E$ is a compact plane set define

$$
A(E, 1)=\left\{f: f \text { analytic on } E^{c}, f(\infty)=0,\|f\|_{L^{\infty}\left(E^{c}\right)} \leq 1\right\}
$$

and define the analytic capacity of $E$ by

$$
\gamma(E)=\sup \left\{\left|f^{\prime}(\infty)\right|: f \in A(E, 1)\right\},
$$

where

$$
f^{\prime}(\infty)=\lim _{z \rightarrow \infty} z f(z) .
$$

If $\gamma(E)=0$, then the only function in $A(E, 1)$ is the constant $f \equiv 0$ and in this case $E$ is removable for bounded analytic functions. For more details about analytic capacity see [G72.

Theorem 1. Let $p>2$, and let $K$ be the four-corner Cantor set $K(p)$. Then $\gamma(K)=0$ but $K$ does not have $\sigma$-finite one-dimensional measure.

Received by the editors April 7, 2000.

2000 Mathematics Subject Classification. Primary 30C20, 28 A75.

The authors were supported in part by NSF Grant DMS-0070782. 
The proof of Theorem 1 depends on a lemma of Jones [J89] used for a proof different from G70] that the $\frac{1}{4}$-Cantor set has zero analytic capacity.

Let $h(t)$ be an increasing continuous function on $t \geq 0$ with $h(0)=0$, and write $\Lambda_{h(t)}(E)$ for the Hausdorff $h$-measure of $E$. Now define an increasing function $h(t)$ so that $h(0)=0$ and $h\left(\sigma_{n}\right)=4^{-n}$ for all $n$. We say $h(t)$ is a measure function corresponding to the Cantor set $K$. For every $n$ define a measure $\mu_{n}$ on $K_{n}$ by $\mu_{n}\left(K_{n, j}\right)=4^{-n}$ for all $j$. Then $\left\{\mu_{n}\right\}$ converges weak-star to a measure $\mu$ supported on $K$ and satisfying $\mu\left(K_{n, j}\right)=4^{-n}$. Suppose $\frac{\sqrt{2}}{2} \sigma_{n} \leq r<\frac{\sqrt{2}}{2} \sigma_{n-1}$ and let $D(z, r)$ be a disk of radius $r$ and center $z \in K$. Then $D(z, r)$ can meet at most 4 squares of sidelength $\sigma_{n}$. Hence

$$
\mu(D(z, r)) \leq 4 \mu\left(K_{n, j}\right)=4 \cdot 4^{-n}=4 h\left(\sigma_{n}\right) \leq 4 h(r)
$$

so that $\mu(D(z, r)) \leq 16 h(r)$ for any disk $D(z, r)$. Therefore $\Lambda_{h}(K)>0$ by Frostman's Theorem G72. Since

$$
\lim _{t \rightarrow 0} \frac{h(t)}{t}=0
$$

if follows that $K$ has non- $\sigma$-finite 1 -dimensional measure.

If $h(t)$ is a measure function corresponding to $K$, then

$$
\int_{0}^{1} \frac{h(t)^{2}}{t^{3}} d t \sim \sum_{n=1}^{\infty} \frac{1}{\left(a_{n}\right)^{2}}=\sum_{n=1}^{\infty} \frac{1}{(\log n)^{\frac{2}{p}}}=\infty .
$$

On the other hand, Mattila [M96] proved that $\gamma(K)>0$ if $K$ is a Cantor set built with squares of side $\sigma_{n}$ and if

$$
\int_{0}^{1} \frac{h(t)^{2}}{t^{3}} d t<\infty
$$

where $h$ is any measure function for corresponding to $K$. Mattila's proof used Menger curvature (see [Me95] and [MMV96]). However, if the Cantor set $K$ has corresponding measure function $h$ satisfying

$$
\int_{0}^{1} \frac{h(t)^{2}}{t^{3}} d t=\infty
$$

then Eiderman [E98] proved that $\gamma^{+}(K)=0$, where

$$
\gamma^{+}(E)=\sup \left\{\int_{E} d \mu:\left|\int_{E} \frac{d \mu(\zeta)}{\zeta-z}\right|<1, \forall z \in \mathbb{C} \backslash E, \mu>0, \operatorname{spt}(\mu) \subset E\right\} .
$$

Since $\gamma^{+}(E) \leq \gamma(E)$, our result is a partial improvement of Eiderman's theorem. Mattila [M96] has conjectured that for Cantor sets of this type $\gamma(K)=0$ if and only if

$$
\int_{0}^{1} \frac{h(t)^{2}}{t^{3}} d t=\infty
$$

when $h$ corresponds to $K$. This latter condition holds if and only if

$$
\sum_{n=1}^{\infty} \frac{1}{\left(a_{n}\right)^{2}}=\infty
$$

If Matilla's conjecture is true, then together with Eiderman's theorem it gives Cantor set evidence supporting the more ambitious conjecture that $\gamma(E)>0$ implies $\gamma^{+}(E)>0$. 
In [G72] it was incorrectly claimed that $\gamma(K)>0$ if and only if

$$
\int_{0}^{1} \frac{h(t)}{t^{2}} d t<\infty
$$

Eiderman, however, found a mistake in the proof. In fact the result in [M96] shows that the claim is false. See L.D. Ivanov [I84] for the first example of a Cantor set of non- $\sigma$-finite linear measure and zero analytic capacity.

\section{Two lemmas of Peter Jones}

We need the following two lemmas from [J89]. The proofs we give are small variations on [J89] and [C90].

Define $\gamma_{j}^{n}=\partial c K_{n, j}$, where $c K_{n, j}$ is the square concentric to $K_{n, j}$ with sidelength $c \sigma_{n}$ and where $c>1$ is chosen so that the $\gamma_{j}^{n}$ do not overlap. We refer to $\gamma_{k}^{m}$ as a square, although it is only the boundary of a square. Notice that

$$
\Lambda_{1}\left(\gamma_{k}^{m}\right)=c \Lambda_{1}\left(\partial K_{m, k}\right)
$$

for the same constant $c$. We associate to each $\gamma_{k}^{m}$ a "square annulus"

$$
A_{k}^{m}=\left\{w: \operatorname{dist}\left(w, \gamma_{k}^{m}\right) \leq \varepsilon_{0} \sigma_{m}\right\}
$$

and we choose $\varepsilon_{0}>0$ so small that the annuli $A_{k}^{m}$ are pairwise disjoint.

Define $\Omega=\overline{\mathbb{C}} \backslash K$. Since $K$ has positive logarithmic capacity, Green's function $G(z, \zeta)$ exists for $\zeta, z \notin K$, and harmonic measure $\omega(\zeta, E)$ exists for $\zeta \notin K$ and $E \subset K$. We write $\omega\left(\zeta, K_{m, k}\right)$ for $\omega\left(\zeta, K_{m, k} \cap K\right)$.

We also define the slightly larger "squares"

$$
S_{m, k}=\left\{w: \operatorname{dist}\left(w, K_{m, k}\right) \leq \varepsilon_{1} \sigma_{m}\right\}
$$

and set

$$
S_{m}=\bigcup_{k=1}^{4^{m}} S_{m, k},
$$

where $\varepsilon_{1}>0$ is so small that $S_{m, k} \cap A_{k}^{m}=\emptyset$. Then $K=\bigcap_{m=1}^{\infty} S_{m}$. Green's function and harmonic measure also exist for the domain $\Omega_{m}=\overline{\bar{C}} \backslash S_{m}$. Denote these by $G_{m}(z, \zeta)$ and $\omega_{m}(\zeta, E)$ respectively.

Lemma 2. Let $z \in A_{k}^{m}$.

(a) There are constants $0<c_{1}<c_{2}<1$, independent of $k$ and $m$, such that

$$
c_{1} \leq \omega_{m}\left(z, \partial S_{m, k}\right) \leq c_{2}
$$

(b) If $\zeta \in \Omega$ and $1 \geq \operatorname{dist}(\zeta, K) \geq 2 \operatorname{dist}(z, K)$, then

$$
G_{m}(z, \zeta) \sim \omega_{m}\left(\zeta, \partial S_{m, k}\right) .
$$

Proof. For $(a)$ note that there is $c^{\prime}>0$ such that there exists a second square annulus $B_{k}^{m}$ so that $A_{k}^{m} \subset B_{k}^{m} \subset \Omega_{m}$ and $\operatorname{dist}\left(z, \partial B_{k}^{m}\right) \geq c^{\prime} \sigma_{m}$. The lower bound then follows by a comparison with $B_{k}^{m}$. There is $S_{m, j}$ with $j \neq k$ such that $\operatorname{dist}\left(S_{m, j}, S_{m, k}\right) \leq c_{4} \sigma_{m}$ and the upper bound follows by a comparison with $\overline{\mathbb{C}} \backslash\left(S_{m, k} \cup S_{m, j}\right)$, using symmetry and Harnack's inequality.

To prove (b) note first that as in the proof of ( $a$ ) there are constants $C_{1}$ and $C_{2}$ such that by Harnack's inequality and a comparison

$$
C_{1} \leq G_{m}(z, w) \leq C_{2}
$$


for $w \in \partial B_{k}^{m}$. Then using the symmetry of Green's function and $(a)$ for a larger square we obtain

$$
C_{1} \omega_{m}\left(\zeta, \partial S_{m, k}\right) \leq G_{m}(\zeta, z) \leq C_{2} \omega_{m}\left(\zeta, \partial S_{m, k}\right) .
$$

We write $\gamma_{k}^{m} \prec \gamma_{j}^{n}$ and say $\gamma_{k}^{m}$ is subordinate to $\gamma_{j}^{n}$ if $\gamma_{j}^{n}$ has winding number one about $\gamma_{k}^{m}$. If the winding number is zero, we write $\gamma_{k}^{m} \nprec \gamma_{j}^{n}$. For any $f \in A(K, 1)$ and $\gamma_{k}^{m}$ define

$$
D\left(\gamma_{k}^{m}\right)=\sup _{w \in \gamma_{k}^{m}}\left|f^{\prime}(w)\right| \sigma_{n}
$$

We say a square $\gamma_{k}^{m}$ has condition $\mathbf{J}$ if

$$
D\left(\gamma_{k}^{m}\right) \leq \delta
$$

for some previously defined $f$ and $\delta>0$.

Lemma 3. Let $f \in A(K, 1)$. For every $\delta>0$ there exists a $C_{0}>0$ such that for every $\gamma_{j}^{n}$ there exists $\gamma_{k}^{m} \prec \gamma_{j}^{n}$ such that $m \leq n+C_{0} \delta^{-2}$ and such that $\gamma_{k}^{m}$ has condition $J$.

Proof. Observe that by Harnack's inequality

$$
\sup _{\gamma_{n, j}}\left|f^{\prime}(z)\right|^{2} \sim \iint_{A_{k}^{n}}\left|f^{\prime}\right|^{2} \frac{d x d y}{\sigma_{n}^{2}} .
$$

Suppose the lemma is false. Choose $\zeta$ with $\operatorname{dist}(\zeta, K)=1$. Then by Green's theorem and the above observation

$$
\begin{aligned}
4 & \geq \int_{\partial \Omega_{n}}|f(z)-f(\zeta)|^{2} d \omega_{n}(\zeta, z) \\
& =\int_{\Omega_{n}}\left|f^{\prime}(z)\right|^{2} G_{n}(z, \zeta) d x d y \\
& \geq \sum_{t=m+1}^{n} \sum_{j} \int_{A_{j}^{t}}\left|f^{\prime}(z)\right|^{2} G_{n}(z, \zeta) d x d y \\
& \geq C \delta^{2} \sum_{t=m+1}^{n} \sum_{j} \omega\left(\zeta, S_{t, j} \cap K\right) \\
& \geq C^{\prime}(n-m) \delta^{2}
\end{aligned}
$$

and we have a contradiction.

\section{A STOPPING-TIME ARGUMENT}

We choose $n_{\delta}=4^{M q}$ where $q>1$ and $M=\left[1+\frac{C_{0}}{\delta^{2}}\right]$. Then because $p>2$ in the definition of $a_{n}=(\log (n+1))^{\frac{1}{p}}$ we have

$$
\lim _{\delta \rightarrow 0^{+}} \delta \cdot a_{n_{\delta} M}=0
$$

and

$$
\lim _{\delta \rightarrow 0^{+}}\left(1-4^{-M}\right)^{n_{\delta}} a_{n_{\delta} M}=0 .
$$

By construction, either $\gamma_{k}^{m} \prec \gamma_{j}^{n}, \gamma_{j}^{n} \prec \gamma_{k}^{m}$, or neither is subordinate to the other. We also write $\gamma_{k}^{m} \nprec F$ if $\gamma_{k}^{m} \nprec \gamma_{j}^{n}$ for all $\gamma_{j}^{n} \in F$ where $F$ is some family of $\gamma_{j}^{n}$. 
Lemma 4. For every $\varepsilon>0$, there exists $\delta>0$, integer $m$ and two families of sets $F_{1}$ and $F_{2}$, such that for some constant $c$ :

(a) $F_{1}=\left\{\gamma_{j}^{n}: \gamma_{j}^{n}\right.$ has condition $\left.J\right\}$,

(b) $\delta \Lambda_{1}\left(\bigcup_{F_{1}} \gamma_{j}^{n}\right)<c \varepsilon$

(c) $F_{2}=\left\{\gamma_{k}^{m}: \gamma_{k}^{m} \nprec F_{1}\right\}$,

(d) $\Lambda_{1}\left(\bigcup_{F_{2}} \gamma_{k}^{m}\right)<c \varepsilon$,

(e) $F_{1} \cup F_{2}$ has winding number 1 about $K$.

Proof. Given $\varepsilon>0$, choose $\delta>0$ so that $\delta a_{n_{\delta} M}<\varepsilon$ and $\left(1-4^{-M}\right)^{n_{\delta}} a_{n_{\delta} M}<\varepsilon$. Fix $m=n_{\delta} M$.

Now define $F_{1}$ to be the set of $\gamma_{k}^{n}$ such that $n \leq m, \gamma_{k}^{n}$ has condition J, and $\gamma_{k}^{n}$ is maximal, i.e. if $K_{n, k} \subset K_{t, j}$ with $t<n$, then $\gamma_{j}^{t}$ does not have condition J. Then $(a),(c)$ and $(e)$ hold for $F_{1}$ and $F_{2}$.

To prove $(b)$ consider $\gamma_{j}^{n} \in F_{1}$. Since $0 \leq n \leq m$ we may replace $\gamma_{j}^{n}$ by $4^{m-n}$ squares of the form $\gamma_{k}^{m}$. Consequently,

$$
\Lambda_{1}\left(\gamma_{j}^{n}\right) \leq 4^{m-n} \cdot \sigma_{m}=4^{-n} a_{m} .
$$

Since the $\gamma_{j}^{n} \in F_{1}$ have pairwise disjoint $K_{n, j}, \bigcup_{F_{1}} \gamma_{j}^{n}$ has smaller $\Lambda_{1}$ measure than $\bigcup_{k=1}^{4^{m}} \gamma_{k}^{m}$ and therefore

$$
\begin{aligned}
\delta \Lambda_{1}\left(\bigcup_{F_{1}} \gamma_{j}^{n}\right) & \leq \delta \Lambda_{1}\left(\bigcup_{k=1}^{4^{m}} \gamma_{k}^{m}\right) \\
& \leq c \delta \cdot 4^{m} \cdot 4^{-m} a_{m} \\
& =c \delta a_{n_{\delta} M} \\
& \leq c \varepsilon,
\end{aligned}
$$

where $c$ is a universal constant.

To prove $(d)$ we use Lemma 3 to obtain

$$
\begin{aligned}
\Lambda_{1}\left(\bigcup_{F_{2}} \gamma_{k}^{m}\right) & \leq c\left(4^{M}-1\right)^{m} 4^{-m} a_{m} \\
& \leq c\left(1-4^{-M}\right)^{n_{\delta}} a_{n_{\delta} M} \\
& \leq c \varepsilon .
\end{aligned}
$$

\section{Proof of Theorem 1}

Suppose $f \in A(K, 1)$ and $\varepsilon>0$ are arbitrary. Let $F_{1}$ and $F_{2}$ be the two families provided by Lemma 4 . Let $z_{k}^{m}$ be an arbitrary point in $\gamma_{k}^{m}$. Then

$$
\begin{aligned}
2 \pi\left|f^{\prime}(\infty)\right| & =\left|\sum_{\gamma_{k}^{m} \in F_{1}} \int_{\gamma_{k}^{m}} f(z) d z+\sum_{\gamma_{k}^{m} \in F_{2}} \int_{\gamma_{k}^{m}} f(z) d z\right| \\
& \leq\left|\sum_{\gamma_{k}^{m} \in F_{1}} \int_{\gamma_{k}^{m}} f(z) d z\right|+\left|\sum_{\gamma_{k}^{m} \in F_{2}} \int_{\gamma_{k}^{m}} f(z) d z\right| \\
& \leq \sum_{\gamma_{k}^{m} \in F_{1}} \int_{\gamma_{k}^{m}}\left|f(z)-f\left(z_{k}^{m}\right)\right| d z+\Lambda_{1}\left(\bigcup_{F_{2}} \gamma_{k}^{m}\right)
\end{aligned}
$$




$$
\begin{aligned}
& \leq c \sum_{\gamma_{k}^{m} \in F_{1}} \int_{\gamma_{k}^{m}} \sup _{w \in \gamma_{k}^{m}}\left|f^{\prime}(w)\right| 4^{-m} a_{m} d z+\varepsilon \\
& =c \sum_{\gamma_{k}^{m} \in F_{1}} D\left(\gamma_{k}^{m}\right) \Lambda_{1}\left(\gamma_{k}^{m}\right)+\varepsilon \\
& \leq c \delta \sum_{\gamma_{k}^{m} \in F_{1}} \Lambda_{1}\left(\gamma_{k}^{m}\right)+\varepsilon \\
& \leq c \delta a_{n_{\delta} M}+\varepsilon \\
& \leq c \varepsilon
\end{aligned}
$$

Since $\varepsilon$ was chosen arbitrarily and $c$ is a universal constant, $f^{\prime}(\infty)=0$. Therefore, $\gamma(K)=0$.

\section{REMARK}

We could obtain a better result if we could improve the estimate in Jones' lemma (Lemma 3). For example, if we could only replace $M=\frac{C_{0}}{\delta^{2}}$ by $\frac{C_{0}}{\delta^{q}}$ for $q<2$, then in the theorem $a_{n}$ could grow like $(\log n)^{\frac{1}{q}}$. As noted above, Mattila [M96] conjectured that $\gamma(K)=0$ if the Cantor set $K$ has $\sum \frac{1}{\left(a_{n}\right)^{2}}=+\infty$. Matilla's conjecture would follow from the method here if the Jones' lemma could be established with $M=c \log \left(\frac{1}{\delta}\right)$ with $c$ constant.

\section{REFERENCES}

[C90] M. Christ, Lectures on Singular Integral Operators, Regional Conference Series in Mathematics 77, American Mathematical Society 1990. MR 92f:42021

[E98] V. Ya. Eiderman, Hausdorff Measure and capacity associated with Cauchy potentials, Translated in Math. Notes (Mat. Zametki.) 1998. MR 99m:28015

[G70] J. Garnett, Positive length but zero analytic capacity, Proc. A.M.S. 24 (1970), 696-699. MR 43:2203

[G72] J. Garnett, Analytic Capacity and Measure, Lecture Notes in Math. 297, SpringerVerlag, 1972. MR 56:12257

[I84] L. D. Ivanov, On sets of analytic capacity zero, Linear and Complex Analysis Problem Book 3, Part II (V.P, Havin, S.V. Kruschev, N.K. Nikolski, ed.) Lecture Notes in Math. 1043, Springer-Verlag, (1984), 498-501. MR 85k:46001

[J89] P. W. Jones, Square functions, Cauchy integrals, analytic capacity and harmonic measure, Harmonic Analysis and Partial Differential Equations (J. Garcia-Cuerva, ed.), Lecture Notes in Math. 1384, Springer-Verlag, (1989), pp. 24-68. MR 91b:42032

[M96] P. Mattila, On the analytic capacity and curvature of some Cantor sets with non- $\sigma$-finite length, Publ. Math. 40 (1996), 195-204. MR 97d:30052

[MMV96] P. Mattila, M. S. Melnikov and J. Verdera, The Cauchy integral, analytic capacity and uniform rectifiability, Ann. of Math. 144 (1996), 127-136. MR 97k:31004

[Me95] M. S. Melnikov, Analytic Capacity: Discrete Approach and curvature of measure, Math. Sb. 186 (1995), 827-846. MR 96f:30020

Department of Mathematics, University of California at Los Angeles, Los Angeles, California 90095

E-mail address: jbg@math.ucla.edu

Department of Mathematics, University of Arizona, Tucson, Arizona 85721

E-mail address: syoshino@math.arizona.edu 